\title{
Capturing, Indexing, Clustering, and Retrieving System History
}

\author{
Ira Cohen ${ }^{1}$, Steve Zhang ${ }^{2}$, Moises Goldszmidt ${ }^{1}$, Julie Symons ${ }^{1}$, Terence Kelly ${ }^{1}$, Armando Fox ${ }^{2}$ \\ 1 \{ira.cohen, moises.goldszmidt, terence.p.kelly, julie.symons\}@ hp.com, Hewlett-Packard Laboratories \\ $2\{$ steveyz,fox\}@cs.stanford.edu, Stanford University
}

\begin{abstract}
In operating today's complex systems, the lack of a systematic way to capture and query the essential system state characterizing an incident of performance failure or unavailability makes it difficult for operators to distinguish recurring problems from new ones, to leverage previous diagnostic efforts, or to establish whether problems seen at different installations of the same site are similar or distinct. We present a method for automatically extracting from a running system an indexable signature that distills the essential system state associated with a problem and can be subjected to clustering and similarity-based retrieval to identify when an observed system state is similar to a previously-observed state. This allows operators to identify and quantify the frequency of recurrent problems, or to leverage problem observations at one site in diagnosing problems at another site. We show that the naive approach to constructing these signatures is ineffective, leading us to a more sophisticated approach based on statistical modeling and inference. Our method requires only that the system's metric of merit as well as a collection of lower-level operational metrics be collected, as is done by existing commercial monitoring tools. Even if the traces have no annotations of prior diagnoses of observed incidents (as is typical), our technique successfully clusters system states corresponding to similar problems, allowing diagnosticians to identify recurring problems or to name the "syndrome" of a group of problems later. We validate our approach on both synthetic traces and several weeks of production traces from a customer-facing geoplexed $24 \times 7$ system; in the latter case, our approach identified a recurring problem that had required extensive manual diagnosis, and also aided the operators in correcting a previous misdiagnosis of a different problem.
\end{abstract}

"Those who cannot remember the past are condemned to repeat it." - George Santayana

\section{Introduction}

When complex software systems misbehave-whether they suffer a partial failure, violate an established servicelevel objective (SLO), or otherwise respond in an unexpected way to workload - understanding the likely causes of the problem can speed repair. While a variety of problems can be solved by simple mechanisms such as rebooting [3], many cannot, including problems related to a misallocation or shortage of resources that leads to a persis- tent performance problem or other anomaly that can only be addressed by a nontrivial configuration change. Understanding and documenting the likely causes of such problems is difficult because they often emerge from the behavior of a collection of low-level metrics such as CPU load, disk I/O rates, etc., and therefore simple "rules of thumb" focusing on a single metric are usually misleading [5].

Furthermore, today there is no systematic way to leverage past diagnostic efforts when a problem arises, even though such efforts may be expensive and are on the critical path of continued system operation. To that end we would like to be able to recognize and retrieve similar problem instances from the past. If the problem was previously resolved, we can try to justify the diagnosis and perhaps even apply the repair actions. Even if the problem remained unresolved, we could gather statistics regarding the frequency or even periodicity of the recurrence of that problem, accumulating necessary information for prioritizing or escalating diagnosis and repair efforts. In order to do these things, we must be able to extract from the system an indexable description that both distills the essential system state associated with the problem and that can be formally manipulated to facilitate automated clustering and similarity based search. Meeting these requirements would enable matching an observed behavior against a database of previously observed ones both for retrieval and determining whether the problem is a recurrent one.

Our contributions are as follows:

1. A formal representation or signature that captures the essential state of an enterprise system and is effective for clustering and similarity based retrieval using known techniques from pattern recognition and information retrieval [6]. We show that the construction of an effective signature is nontrivial - the naive approach yields poor clustering and retrieval behaviors, but good results are obtained with an approach based on our prior successful use of statistical methods to capture relationships between low-level system metrics and high-level behaviors [22, 5].

2. The use of this representation to cluster and identify performance problems, and compute statistics about the frequency of their occurrence. This in turn lets an operator distinguish a recurrent condition from a transient or first-time condition, and even annotate the corresponding signature(s) with a repair proce- 
dure or other explanation for future reference when the same problem recurs.

3. A demonstration of how the representation and clustering can be used across different sites to aid diagnosis.

Our experimental validation is conducted on a realistic testbed with injected performance faults, and on production traces from several weeks of operation of a real customer-facing Web application in our organization.

The rest of the paper is organized as follows. Section 2 outlines our approach and methodology and provides some background on the statistical modeling technique used. Section 3 describes both our controlled testbed and the production traces used in our experiments. Section 4 presents our results. Specifically, Section 4.1 compares three methods of signature construction. Section 4.2 illustrates the use of our method for identifying recurrent problems in a real production environment. Section 4.3 shows that signatures can be leveraged across sites. In Section 5 we review related work. We discuss some caveats and ramifications in Section 6 and offer concluding remarks in Section 7.

\section{Problem Statement, Approach, and Methodology}

We address three problems:

1. Signature construction: What representation should we use to capture the essentials of the system state and enable clustering (grouping) and retrieval?

2. Discovery and exploration: How do we facilitate the identification of recurrent issues and the retrieval of similar problems?

3. Evaluation methodology:

(a) How can we determine that our signatures are indeed capturing the system state, that is, that the information contained in them effectively serves as a "fingerprint" of a high-level system condition of interest such as a performance anomaly?

(b) How can we verify that clustering (based on these signatures) is meaningful, that is, that signatures that are similar according to some similarity metric are fingerprints of problems whose diagnoses are similar?

(c) How can we evaluate the quality of retrieval, that is, how can we verify that a query to retrieve similar signatures is returning a high percentage of actual matches with a low false positive rate?

The evaluation in particular has high practical importance: since our intent is to facilitate the exploration of the past history of the system to identify recurrent problems and similar situations in different systems, the users of our technology (system operators) must be confident that problem instances reported as belonging to the same group or cluster are indeed related. This is why our evaluation criteria are defined operationally, e.g., to say that clustering is "meaningful" is to say that similar signatures do indeed identify problems with similar root-cause diagnoses in practice.

Without loss of generality, when we refer to an "Internet service" in the following discussion, we mean an externalrequest-driven, interactive application based on the standard three-tier architecture [7] of a stateless Web server, a tier containing application logic possibly running on top of a middleware platform, and a persistence tier containing one or more databases.

\subsection{Sketch of the Approach}

We assume the system's operational policy defines one or more reference metrics (average response time, request throughput, etc.) and a threshold on each of these metrics (or a threshold over a collection of metrics). These reference metrics and the thresholds define a service level objective or SLO. We say the system is in violation of its SLO if the metric(s) exceed the policy threshold, and in compliance with its SLO otherwise. The SLO may be defined as an aggregate, for example, "at least $60 \%$ of requests during a 5-minute interval have a response time

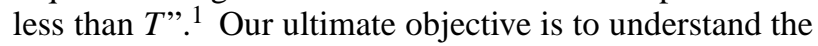
underlying causes of high-level system behavior and how these causes manifest as SLO violation or compliance. We concentrate on the questions stated above of identifying recurrent performance issues, and the automatic retrieval of similar problems.

We begin by evaluating several candidates for representations of the essentials of the system state, which we call signatures. We then evaluate the use of automated clustering [6] for grouping SLO violations in terms of their signatures, identify recurrent problems, and expose collections of metrics that together can become a syndrome of a performance problem. We then evaluate information retrieval techniques for finding signatures based on similarity [20]. This ability will enable an operator to search databases indexed with signatures and find past occurrences of similar problems. This in turn will result in the operator leveraging past diagnostics and repair efforts.

In our evaluation we will use data from traces collected from both a realistic testbed and workload, and from a production system that has suffered several SLO violations over a period of three months. In the case of the testbed we run the system and periodically inject specific faults in order to trigger SLO violations. The testbed enables us to annotate each SLO violation with its root cause, providing ground truth for verifying the results of the automated clustering and of similarity retrieval. For the real

\footnotetext{
${ }^{1}$ Note that even SLO's expressed in terms of performance are deeply connected to availability, because availability problems often manifest early as performance problems and because understanding how different parts of the system affect availability is a similar problem to understanding how different parts of the system affect high-level performance.
} 
application, we have annotations for only a subset of the instances of SLO violations, and therefore it is only in these instances that we will verify the use of information retrieval techniques. We will define in Section 2.3 a notion called purity that will enable us to evaluate the use clustering even in cases with partial annotations.

Note that whether annotations are available or not, clustering enables us to group SLO violations in terms of similar signatures, and discover different types of SLO violation instances, recurrent problems, etc. Similarly, retrieval enables us to find and leverage past diagnoses and repairs. For example, as we report in Sections 4.2 and 4.3 we find that if the operators of the production system had had access to our technology, a problem that was initially identified as unique but later found to be a recurrence of a prior problem could have been immediately identified as such. Also, one incident that was initially classified as a recurring problem exhibited a very dissimilar signature (using our method) than the alleged original problem; manual rediagnosis showed that our method was correct, and indeed the second problem was not a repeated manifestation of the first problem.

\subsection{Signatures: Capturing System State}

The first issue we address is that of a representation that captures those aspects of system state that serve as a "fingerprint" of a particular system condition. Our goal is to capture the essential system state that contributes to SLO violation or compliance, and to do so using a representation that provides information useful in the diagnosis of this state, in clustering (grouping) of this state (with similar states), and in the retrieval process. We will call such a representation a signature of the system state. We make the following assumptions:

1. We assume that we can continuously measure whether the system is in violation or compliance at any given time with respect to the SLO. This can be done, for example, by examining server logs containing timing information or by running probes.

2 . We assume that we can continuously measure a collection of metrics that characterize the "low level" operation of the system, such as CPU utilization, queue lengths, I/O latencies, etc. This information can come from OS facilities, from commercial operations tools such as HP OpenView, from instrumented middleware (as was done in [4]), from server logs, etc.

Since we can by assumption collect low-level system metrics, it would seem reasonable as a starting point to simply use these raw values as the signature. As will be seen in Section 4.1, our experiments allow us to conclude that signatures based on using raw values are not as effective as an approach that builds on our prior work on metric attribution $[22,5]$. In that work we automatically build models that identify the set of low-level system and application metrics that correlate with each particular instance of the SLO state. We hypothesize that this attribution information is the key to constructing signatures that correctly characterize and distinguish different causes of SLO violations. We therefore spend the rest of this section reviewing the relevant aspects of that work and how it relates to signature construction.

The metric attribution process goes as follows. The input is a data $\log$ containing vectors $\vec{M}$ of low-level system and application metrics and the state $Y$ (compliance or violation) of the system. We divide time into regular epochs (e.g., five-minute intervals) and we have one such vector for each epoch. Each element $m_{i}$ of the $\vec{M}$ for an epoch contains the value of the specific metric, an average over the epoch, and $Y$ contains a discrete value depending on whether the SLO was violated or not. Relying on pattern classification techniques and probabilistic modeling, the algorithms in $[22,5]$ yield as output an ensemble of probabilistic models characterizing the behavior of the metrics during intervals of both compliance and violation.

Each one of these models essentially represents the relationship between the metrics and the SLO state as a joint probability distribution. We use the Tree-Augmented Naive Bayes models (TAN) to represent the joint probability distribution. Out of this distribution we can extract a characterization of each metric and its contribution to the SLO state. Let the term $P\left(m_{i} \mid m_{p_{i}}, s^{-}\right)$represent the resulting probabilistic model for metric $m_{i}$ under violations $\left(s^{-}\right)$, and let $P\left(m_{i} \mid m_{p_{i}}, s^{+}\right)$, represent a probabilistic model for the same metric under an SLO state of compliance. ${ }^{2}$ Using these models we can identify for a given instance of $\vec{M}$, which metrics (because of their values) are more likely to come from their characteristic distribution during violation. This process is called metric attribution. Formally, for a given instance, a metric is flagged as "attributable" if:

$$
\log P\left(m_{i} \mid m_{p_{i}}, s^{-}\right)>\log P\left(m_{i} \mid m_{p_{i}}, s^{+}\right),
$$

that is, $m_{i}$ 's value is closer to its characteristic value for the "violation" distribution than to its characteristic value for the "compliance" distribution. The interpretation is that the observed value of $m_{i}$ is more likely to come from the distribution where the SLO state $Y$ is that of violation. In addition, the methods in $[5,22]$ are able to identify metrics that yield no information on the SLO state. We call such metrics irrelevant.

The process of constructing signatures is as follows. Given a system trace, we follow the procedure detailed in [22] to continuously learn an ensemble of models; for every epoch, a relevant (to the particular SLO state) subset of models from the ensemble is selected; from those we extract a list of metrics whose values are "abnormal" (resp. "normal"), that is, the values are identified as being closer to their characteristic values during periods of SLO

\footnotetext{
${ }^{2}$ The term $m_{p_{i}}$ represents a metric directly correlated with metric $m_{i}$ in the Bayesian network representation. Interested readers should consult [5].
} 
violation (resp. compliance). ${ }^{3}$ We then extract the signatures, by yielding a transformed data log containing a set of vectors $\vec{S}$ where:

- entry $s_{i}=1$ if that metric is selected by the relevant models whose value is determined to be abnormal. We say the metric is attributed;

- entry $s_{i}=-1$ if the metric is selected by relevant models but is not found to be "abnormal". We say that the metric is not attributed;

- entry $s_{i}=0$ if the metric is not selected by any of the models at all-we say that the metric is irrelevant to the SLO state.

Each vector $\vec{S}$ is then a signature for that epoch. Note that we produce a signature for every epoch including those in which the SLO was not violated. The intended semantics of a signature are as follows. A metric receives a value of 1 if its raw value is more likely to come from the distribution of values associated with SLO violation (i.e., Inequality 1 is true). These semantics holds for both periods of violation and compliance. As will become apparent, these signatures are useful both for characterization of the behavior of the system in compliance periods, and in violation periods.

Thus, given system traces collected on an Internet service, our approach generates a database consisting of the signatures describing the system state, coupled with the SLO state.

\subsection{Clustering of Signatures}

The objective when applying clustering to a database of signatures is to find the natural groupings or clusters of these signatures that characterize the different performance problems and normal operation regimes. The output of the clustering is a set of clusters, plus a characterization of the cluster center. By inspecting the actual elements of the signature database in each cluster we can identify different regions of normality as well as recurrent problems. In addition, the centroid of a cluster of problem behaviors can be used as a syndrome for the problem, since it highlights the metrics that are in a sense characteristic of a set of manifestations of the same problem.

In order to render the description above operational, we need to specify a distance metric and a clustering algorithm that minimizes the distortion (sum of distances of each signature to its nearest cluster center). In this paper we explored both the $L_{1}$ and $L_{2}$ norms as distance metrics $\left(L_{p}=\sqrt[p]{\sum_{i}\left|x_{1}^{i}-x_{2}^{i}\right| p}\right)$. As our basic clustering algorithm we will use the standard iterative algorithms [6]: k-medians for the $L_{1}$ norm, and k-means for the $L_{2}$ norm. These algorithms find k cluster centers (medians and mean respectively) that minimize the distortion

\footnotetext{
${ }^{3}$ Mechanism for fusing the information from different models are described in detail in [22].
}

as defined above. We will report only on the results coming from the $L_{1}$ norm. Although the results for the $L_{2}$ norm were quantitatively different, they were qualitatively similar and offered no new insight as far as making decisions about the signature representation. In addition the centers of the clusters in the k-medians case are represented by actual signatures that are members of the cluster. ${ }^{4}$

Ideally, we would like each cluster to contain signatures belonging to a single class of performance problems (SLO violations), or else signatures belonging only to periods of SLO compliance. We introduce a score determining the purity of a clustering to formalize this intuition. In the case where we have no annotations, we distinguish signatures only in terms of their corresponding SLO state (compliance or violation). We count the number of signatures in each cluster that describe epochs of compliance and the number that describe epochs of violations. These counts are then normalized by the total number of signatures (the sum of the two), to produce probability estimates $p_{c}$ and $p_{v}\left(p_{c}+p_{v}=1\right)$. This are used in turn to score "purity" of each cluster. A cluster is pure if it contains signatures of only compliance or only violation epochs, i.e., if either $p_{c}$ or $p_{v}$ are 1 . With these probabilities, we compute the entropy of each cluster, given as: $H=-p_{c} \log _{2}\left(p_{c}\right)-p_{v} \log _{2}\left(p_{v}\right)$. For a pure cluster, entropy is 0 , which is the best result. The entropy is 1 when the cluster is evenly split between the two types of signatures $\left(p_{c}=p_{v}=0.5\right)$. It is straightforward to generalize the definition of purity score when there are labels distinguishing different annotations corresponding to SLO violations.

We compute the overall average entropy of the all of the clusters weighted by the normalized cluster size to give us a measure of purity of the clustering result. Average entropy close to 0 is a strong indication that indeed the signatures captured meaningful characteristics of the periods of violations in contrast to periods of non-violation. Indeed, we will use the purity score to compare different proposals for generating signatures (see Figures 2, and 3).

In order to include the purity information in the clustering process, we added an iterative loop using the standard k-medians (k-means) as a building block. ${ }^{5}$ In the first step k-medians is applied to the whole database $D$ of signatures, yielding $k$ clusters as its output. The purity score is applied to each one of these clusters. If the score is above an input threshold $t_{p}$, then the procedure stops. Otherwise, k-medians is applied once more to the signatures for those clusters that didn't pass the test. There are two parameters used to control these process: a number $k$ which is the maximum of clusters expected in each application of $k$-medians, and the total $k_{t o t}$ of clusters expected. The process stops when $k_{t o t}$ is reached. There are a number of procedures for determining these parameters, in-

\footnotetext{
${ }^{4} \mathrm{~A}$ complete analysis of these norms is well beyond the scope of this paper and will be explored in future work.

${ }^{5}$ In the rest of the description we will only mention k-medians with the understanding that when the $L_{2}$ norm is used the actual algorithm is k-means.
} 
cluding score metrics with regularization components and search procedures which increase their value gradually until no significant improvement in the clustering distortion is achieved [6]. In this paper we explore the space of $k_{t o t}$ and set $k=5$. These values where appropriate for evaluating the power of the signature extraction process and displaying its properties. We also checked the amount by which our algorithm will affect the minimization in distortion with regards to a straightforward application of the $\mathrm{k}$-medians algorithm and found it to be negligible.

When we compare different candidate methods of signature construction in section 4.1, we will use the purity of the clustering process as providing evidence of the information contained in each approach. Signatures that enable clusterings with a higher degree of purity (lower entropy) will be favored as they clearly contain enough information to enable the automated grouping of the different SLO related states (see Section 4.1.1).

\subsection{Retrieval of Signatures}

Using information retrieval techniques we can retrieve from a database all previous instances that are "similar" to a specific signature $S$. This capability enables us to leverage past diagnosis and repairs and in general all information about previous instances displaying similar characteristics (as captured in the signature vector).

As in the case of clustering, similarity is formalized in the context of a distance metric, and as in that case, we explored both $L_{1}$ and $L_{2}$ norms, displaying only the results for the $L_{1}$ norm.

Our evaluation will follow the standard measures from the machine learning and information retrieval community [20]. The evaluation focusses on the quality of the retrieval with respect to the similarity and the quality of the signatures. If a specific annotation $A$ is associated with a (set of) signature(s) $S$ we expect that when similar signatures are used, the retrieval process yields signatures also associated with annotation $A$. Therefore, in evaluating our retrieval results, we consider only those signatures associated with annotations (see Sections 3.1 and 3.3).

Formally the process of retrieval proceeds as follows: given a signature, return the $N$ closest signatures to it from the existing signature database. Given known annotations both to the query signature and the signatures in the database, we compute the two standard measures of retrieval quality: Precision and Recall.

For a given query, precision measures what fraction of the $N$ returned items have the matching annotation (1.0 is perfect); recall measures the percentage of signatures in the database with the same annotation as the query that are actually retrieved. Note that the maximal value of 1.0 is achieved only when $N$ is at least equal to the number of signatures with the same annotation as the query signature. As $N$ increases recall goes up but precision typically goes down, as it becomes harder to retrieve only signatures that have a matching annotation.
Following the common practice in the information retrieval community, we increase $N$ and measure the precision/recall pair, until we achieve a recall of 1.0. We then plot precision as a function of recall, to produce the Precision-Recall curve (see Figure 4 for an example). A perfect precision/recall curve has precision of 1.0 for all values of recall. As in the case of clustering, we will use precision and recall to evaluate the different proposals for a signature (see Section 4.1.2).

\section{Trace Collection and Characterization}

Our empirical results are based on large and detailed traces collected from two distributed applications, one serving synthetic workloads in a controlled laboratory environment and the other serving real customers in a globallydistributed production environment. These two traces allow us to validate our methods in complementary ways. The testbed trace is annotated with known root causes of performance problems. Annotated data allows us to evaluate our signature-based diagnostic methods in terms of simple information-retrieval performance measures (e.g., precision, recall). The production trace is not annotated as well or as thoroughly as the testbed trace. By treating it as an unlabeled data set, we can use it to evaluate the effectiveness of the signature clustering, which in turn provides identification of the "syndromes" describing performance problems. Since we do have annotations to some of the signatures in the production trace, we can further validate the accuracy of our retrieval on real-world data.

Our traces record two kinds of data about each application: application-level performance data for our models' SLO indicator, and system-level resource utilization metrics (e.g., CPU utilization). Our tools measure the latter as averages in non-overlapping windows.

\subsection{Testbed Traces}

Our controlled experiments use the popular PetStore ecommerce sample application, which implements an electronic storefront with browsing, shopping cart and checkout. Each tier (Web, J2EE, database) runs on a separate HP NetServer LPr server (500 MHz Pentium II, 512 MB RAM, 9 GB disk, 100 Mbps network cards, Windows 2000 Server SP4) connected by a switched 100 Mbps full-duplex network. Apache's extended HTTPD log format provides us with per-transaction response times and we obtain system-level metrics from HP OpenView Operations Agent running on each host. A detailed description of our testbed's hardware, software, networking, and workload generation is available in [22]. We collect 62 individual metrics at 15 -second intervals and aggregated to one-minute windows containing their means and variances. We pre-process our raw measurements from the Apache logs to average transaction response times over the same windows and then join all data 
from the same application into a single trace for subsequent analysis.

We use the standard load generator httperf [11] to generate workloads in which simulated clients enter the site, browse merchandise, add items to a shopping cart, and checkout, with tunable probabilities governing the transition from "browse item" to "add item to cart" (probability $P_{b}$ ) and from "add item to cart" to "checkout cart" (probability $P_{c}$ ). We measure the average response time of client requests in each 1 minute window and require that the average response time stay below $100 \mathrm{msec}$ to maintain SLO compliance. We created three handcrafted fault loads designed to cause SLO violations. In the first, we alternate one-hour periods of $P_{b}=P_{c}=0.7$ with one-hour periods of $P_{b}=P_{c}=1.0$ (a "BuySpree"). In the second, we execute a parasitic program on the database server machine that consumes about $30 \%$ of the available CPU during alternating one hour intervals, but no other major resources ("DBCPU contention"). The third faultload does the same thing but on the application server rather than the database server ("APPCPU contention").

Note that these faultloads simulate both faults due to internal problems (CPU contention) and faults resulting from changes in workload (extreme buying plateaus during BuySpree). In both cases, the injected faults correspond to annotations of known root causes of performance problems in the final processed trace that we employ in our analyses and evaluations.

\subsection{Production System Traces}

Our second trace is based on measurements collected at several key points in a globally-distributed application that we call "FT" for confidentiality reasons. FT serves business-critical customers on six continents 24 hours per day, 365 days per year. Its system architecture therefore incorporates redundancy and failover features both locally and globally, as shown in Figure 1. Table 1 summarizes key hardware and software components in FT, and the transaction volumes recorded by our traces (Table 2) demonstrate the non-trivial workloads of the FT installations. All hosts at the application server and database server tiers are HP 9000/800 servers running the HPUX B.11.11 operating system, except that one database server in Asia is an HP rp7410 server.

HP OpenView Performance Agent (OVPA) provides system utilization metrics for application server and database hosts. FT is instrumented at the application level with ARM [18], providing transaction response times. OVPA and ARM data are aggregated into 5-minute windows in the processed traces we analyze. We have traces from the Americas and Asia/Pacific hubs but not from Europe. Table 2 summarizes our FT traces.

The service-level agreement associated with FT defines different response time SLOs for different transaction types. Compliance is measured on every transaction and counts are aggregated in 5-minute buckets. Our cri-

$\begin{array}{lrcccc}\text { Region } & \text { Role } & \begin{array}{c}\# \\ \text { hosts }\end{array} & \begin{array}{c}\# \\ \text { CPUs }\end{array} & \begin{array}{c}\# \\ \text { disks }\end{array} & \text { RAM } \\ \text { (GB) }\end{array}$

Table 1: Key hardware and software components in FT. The two app server hosts in Asia have different numbers of disks. All app servers ran WebLogic and all DB servers ran Oracle 9i. Most of the DB servers had $550 \mathrm{MHz}$ CPUs.

\begin{tabular}{|c|c|c|c|c|c|}
\hline \multirow[b]{2}{*}{ Server } & \multirow[b]{2}{*}{ Dates } & \multicolumn{3}{|c|}{ transactions/min } & \multirow{2}{*}{$\begin{array}{r}\% \text { SLO } \\
\text { viol }\end{array}$} \\
\hline & & mean & $95 \%$ & $\max$ & \\
\hline AM1 & $12 / 14-1 / 14$ & 208.6 & 456.4 & $1,387.2$ & 23 \\
\hline AM2 & $12 / 13-2 / 08$ & 207.9 & 458.0 & 977.4 & 20 \\
\hline AP1 & $12 / 17-1 / 05$ & 39.9 & 118.2 & 458.4 & \\
\hline AP2 & $12 / 17-1 / 30$ & 52.1 & 172.8 & 775.0 & \\
\hline
\end{tabular}

Table 2: Summary of FT application traces. The last column is the percentage of transactions which violated their SLO in the data. Trace collection began in late 2004 and ended in early 2005. "AM" and "AP" servers were located in the Americas and Asia, respectively.

terion for SLO violation is whether more than $40 \%$ of the transactions in a 5-minute window violated their transaction SLO.

FT is well suited to our interests because its requirements include high performance as well as high availability. Furthermore performance debugging in FT is particularly challenging for two reasons. First, different organizations are responsible for FT itself and for the application server infrastructure in which crucial FT components run (the latter is delimited with a shaded dashed oval in Figure 1); opportunities for inter-organizational finger-pointing abound when end-to-end performance is poor. Second, FT's supporting infrastructure is physically partitioned into three regions with separate operational staffs. A performance problem that occurs in the afternoon in each region, for example, will occur three different times, will appear to be specific to a single region each time it occurs, and will demand the attention of three separate teams of system operators. Cost-effective performance debugging in FT requires that commonalities be recognized across regions, across time, and across organizational boundaries, and that different teams of human diagnosticians leverage one another's efforts.

Another attractive feature of our FT traces is that some of our 5-minute samples are annotated, in the sense that they correspond to times when we know that a specific performance problem occurred whose root cause was subsequently diagnosed. In the next section we describe this 


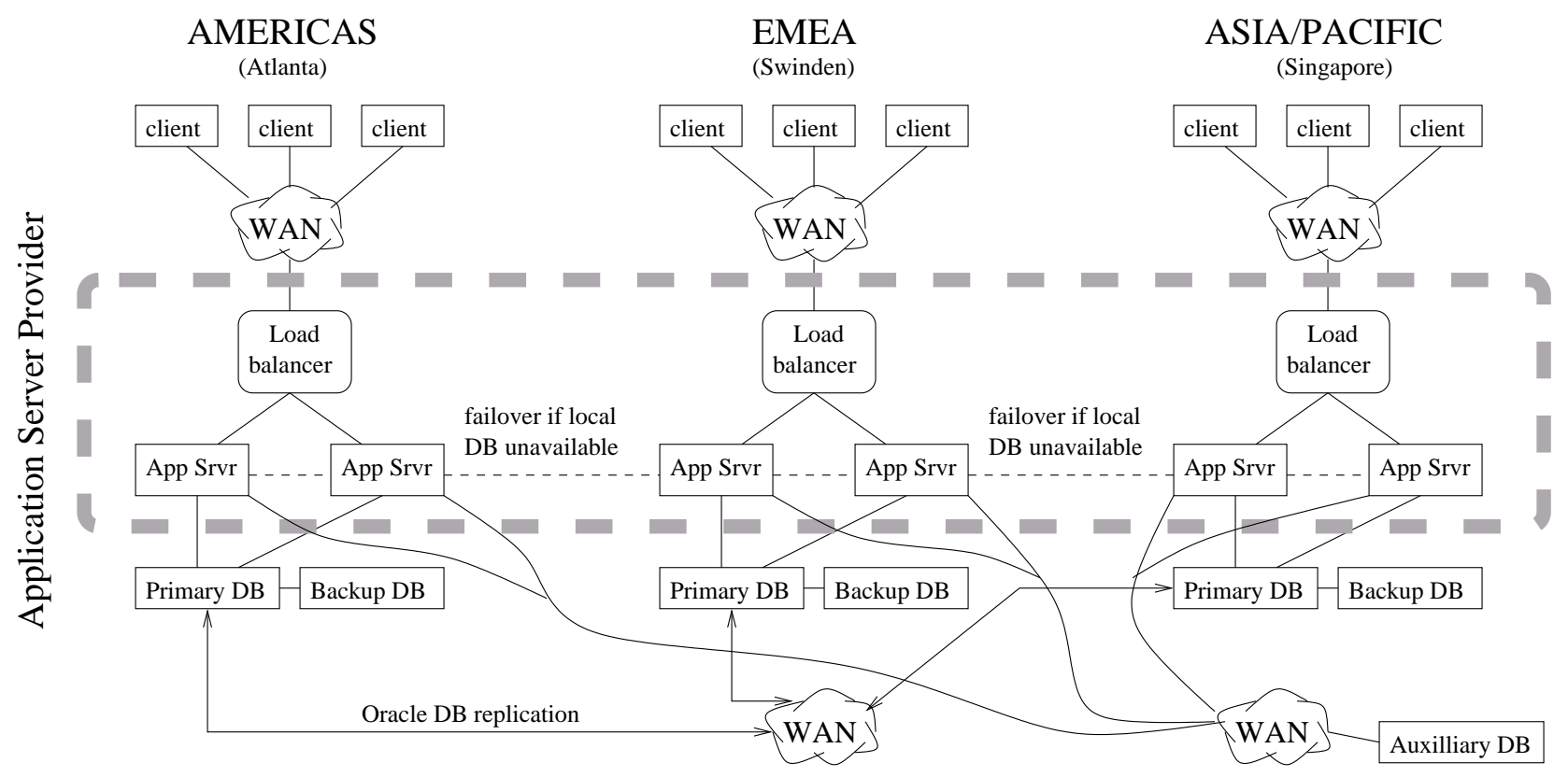

Figure 1: Architecture of the "FT" production system. FT is a globally-distributed multi-tiered application with regional hubs in the Americas, Europe/Middle East/Africa, and Asia. Different organizations are responsible for the FT application and the application server on which it runs; the latter is indicated by a shaded dashed oval in the figure. FT has a globally-distributed main database and an additional auxiliary databases, managed by a third organization, shown in the lower right.

problem, which illustrates both the challenges that we face and the opportunities that our approach attempts to exploit.

\subsection{A Diagnosed Problem: Insufficient Database Connections (IDC)}

The FT production system experienced a recurrent performance problem mainly in the Americas domain during December 2004 and January 2005. During episodes of this problem, business-critical customers experienced latencies of several minutes on transactions that normally complete within seconds. The operators who first detected the problem described it as "stuck threads" in the application server because WebLogic issued messages in a logfile each time it diagnosed a stuck thread. There can be many causes for threads to become stuck, therefore it is necessary to look for other symptoms to diagnose the cause.

Due to the severity of the problem, a joint task force comprising both FT application developers and application server administrators quickly formed to address it. This team eventually diagnosed and repaired the root cause of the performance problem, thus providing annotations for data points in our traces corresponding to episodes. Our account of the problem is based on detailed bug-tracking database entries and e-mail correspondence among the troubleshooters.

After several weeks of debugging, the problem was traced to a too-small pool of database connections. Under heavy load, application threads sometimes had to wait more than 600 seconds to acquire a connection, and were therefore flagged as "stuck threads" by WebLogic. The problem was solved by increasing the connection pool size by $25 \%$. We use the annotation IDC (Insufficient Database Connections) to refer to this problem.

\section{Results}

To substantiate our claims, we perform analysis on the data collected from both the experimental testbed excited with synthetic workloads and from the globallydistributed production environment described in Section 3. We will use ETB (for Experimental TestBed) to identify traces in the first case and RWA (Real World Application) for the second case. For the ETB experiments, we intentionally injected known faults into the system to cause performance problems. The ETB trace is therefore reliably annotated with the appropriate fault per epoch. In the RWA we have only partial annotations, related to one diagnosed problem, as described in Section 3.3. These annotations as well as the fact that we have an objective measure of performance as reflected by the SLO state of compliance and violation will provide ground truth against which we can compare the results of the clustering and retrieval operations. 


\begin{tabular}{|l|c|c|c|}
\hline Metric & $\begin{array}{c}\text { Raw } \\
\text { Value }\end{array}$ & Attr. & $\begin{array}{c}\text { Raw Value } \\
\text { \& Attr }\end{array}$ \\
\hline transaction count & 398.00 & 0 & 0 \\
gbl app cpu util & 97.47 & 1 & 97.47 \\
gbl app alive proc & 449 & 0 & 0 \\
gbl app active proc & 357 & 0 & 0 \\
gbl app run queue & 10.57 & 1 & 10.57 \\
gbl app net in packet rate & 817 & 1 & 817 \\
gbl mem util & 54.62 & 1 & 54.62 \\
gbl mem user util & 26.32 & 1 & 26.32 \\
DB1 CPU util & 25.96 & -1 & -25.96 \\
\hline
\end{tabular}

Table 3: Examples of the different signature proposals, showing a subset of the metrics collected in the production environment. The first column is of raw values (not normalized to preserve the context of these metrics), second is metric attribution (with possible values in $\{+1,0,-1\}$ ), and third is the AND of raw values and metric attribution.

\subsection{Claim 1: Evaluating Proposals for Sig- natures}

We evaluate and compare three possible approaches for creating signatures. Let $\vec{S}$ denote the vector representing the signature. In all cases the elements $s_{i}$ in this vector correspond to a specific system, application, or workload metric.

1. Raw values: in this case we represent a signature $\vec{S}$ using the raw values of the metrics. In other words $\vec{S}=\vec{M}$. Following common practice in data analysis [6], we normalize these values to $[0,1]$ to prevent scaling issues from influencing similarity metrics and clustering. ${ }^{6}$ This signature is the most naive and requires no extra processing of the traces.

2. Metric attribution: If the attribution as described in Section 2.2 flags the metric $m_{i}$ to be attributable, then $s_{i}=1$, otherwise $s_{i}=-1$. If the metric $m_{i}$ is not even considered by the models for the attribution process, then the metric is considered to be irrelevant to the SLO state and $s_{i}=0$. This requires significant computation [5], but we showed in [22] that the computations could be done on millisecond timescales, allowing this approach to be used in real time.

3. Metric attribution and raw values: This is similar to the previous approach except that the raw value of the metric is multiplied by its entry $s_{i}$ as explained in the previous item. ${ }^{7}$.

We evaluate signature-generation approaches based on the quality of clustering and retrieval operations. We demonstrate that signatures based on metric attribution are superior for our purposes. This strongly implies that metric attribution captures information about system state that goes beyond the raw values of the collected metrics, further validating the results in $[22,5]$.

\footnotetext{
${ }^{6}$ The results with unnormalized values were much worse for the signatures relying on the raw values.

${ }^{7}$ The intuition is that the information contained in the value of the metric is added to the information in the attribution process
}

We also remark that in our experiments we see that some metrics are consistently deemed irrelevant for all time epochs in the traces (e.g., in the RWA data the root $\mathrm{CPU}$, memory and disk utilization on the application server were never found to be relevant by any model, getting a value of 0 for all epochs). In some cases such an observation can lead to reducing the amount of metrics being collected, although that loss of information can be detrimental in cases where a dropped metric becomes relevant in future performance problems. In addition, because our modeling process rapidly narrows down to a small number of metrics that are highly correlated with the SLO state, unless the expense of data collection is significant, we discourage removing any metrics from the measurement apparatus.

\subsubsection{Signatures and Clustering}

We perform a clustering of the signatures as described in Section 2.3. To evaluate the quality of the clusters, we rely on the notion of purity also described in the same section. Entropy is used as a measure of purity with low entropy implying that the clusters contain only one type of annotated value (and hence are of a better quality). In the case of the ETB we have four annotations: one refers to periods of SLO compliance and the other three are given by the cause related to the injected problem. For the RWA, we use SLO compliance and violation to check purity. In both cases we vary the number of clusters and check the variation in the weighted average entropy over the clusters. Stable entropy across different cluster numbers is an indication of the robustness of the clustering output. Figures 2 and 3 show the resultant entropy for each signature generation method and number of clusters. Note that in both cases, ETB and RWA, the clustering using attribution information clearly yields purer clusters than the clustering using only the raw value of the metrics.

Table 4 provides the counts of each annotation with 9 clusters using signatures based on metric attribution on one of the Americas production systems. Presenting the raw counts in a cluster provides the application administrator a sense of the intensity of the pure clusters, giving her the capability to focus attention on the more prominent clusters of performance problem instances. It is worth noting that one of the clusters (cluster 3 ) contained most of the instances of the IDC problem (75\%), which is significant since the clustering algorithm did not have knowledge of the IDC annotation of these instances, but only knew that these were violation instances. Cluster 9 and 10 indicate that for about $10 \%$ of the data the signatures were not able to separate between periods of violation and compliance.

\subsubsection{Signatures and Retrieval}

Figure 4 shows precision/recall curves of retrieval exercises performed on the ETB traces using the three approaches described above for the signatures (see Sec- 


\begin{tabular}{|c|c|c|c|}
\hline Cluster \# & \# vio & \# compl & Entropy \\
\hline 1 & 552 & 27 & 0.27 \\
\hline 2 & 225 & 0 & 0.00 \\
\hline 3 & 265 & 2 & 0.06 \\
\hline 4 & 0 & 1304 & 0.00 \\
\hline 5 & 1 & 1557 & 0.00 \\
\hline 6 & 0 & 1555 & 0.00 \\
\hline 7 & 0 & 1302 & 0.00 \\
\hline 8 & 216 & 274 & 0.99 \\
\hline 9 & 100 & 128 & 0.99 \\
\hline
\end{tabular}

Table 4: Example of a clustering instance using signatures based on metric attribution. The first column is a count of number of violation instances, the second shows the number of compliance instances, and the third shows the cluster entropy based on the purity of the cluster. Cluster 3 contains almost all the instances corresponding to the IDC problem; 201 instances of the 265 violation instances in cluster 3 are annotated as the IDC problem, the remainder 68 IDC annotated instances are distributed between cluster 1 and 2.

tion 2.4 for the definition of these curves). Precision/recall performance is better in direct proportion to the area under its curve. Clearly the use of attribution provides an informational advantage, as the curves using attribution are far superior.

Our real-world traces were collected during a period when a misconfiguration was causing a performance problem (the "IDC" problem discussed in Section 3). Overall, 269 epochs were annotated with this problem. Figure 5 shows the location of these epochs over the month long trace, overlayed on the value of the reference metric (transactions average response time). It can be seen that the issue occurred intermittently over that period.

Figure 6 shows the precision-recall graphs for retrieving signatures of the IDC problem. The graph shows that high precision is achieved for a relatively large range of the recall value. For example, of the top 100 signatures retrieved, 92 are correctly retrieved, leading to a precision of $92 \%$ (and recall of $34.2 \%$ ). Such a precision would be more than sufficient for an operator to safely infer that the label attached to the majority of signatures being retrieved matches the problem described by the query signature. As in the case of clustering, these results confirm that the use of attribution in the generation of signatures provides the required information.

\subsection{Claim 2: Identifying Recurrent Prob- lems}

We show that the clustering is meaningful: each cluster groups signatures that would have the same annotations if they were provided and the centroids can be used as the syndrome that characterizes that group of signatures. Therefore clustering can be used to identify recurrent is-

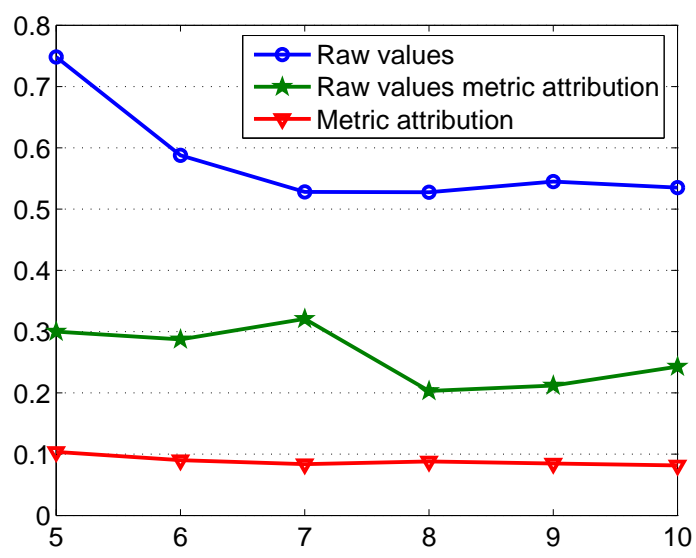

Figure 2: Clustering on the annotated data from ETB. The signature method relying on attribution performs best and is more stable across different number of clusters. $\mathrm{X}$ axis denotes the number of clusters.

sues and to gather statistics regarding their frequency and periodicity.

First, based on the results of the Subsection 4.1 we can assert that the clustering as applied to the data in the RWA is robust. As Figure 3 indicates, the entropy of the clusters does not change significantly as we increase the number of clusters, implying that the existing clusters are being subdivided rather than completely new ones created. Recall that the entropy is an indication of the "purity" of the cluster, namely of the elements in the cluster what percentage belong to the same annotation.

Second, we demonstrate that the clustering is meaningful for the case of nine clusters. Table 4 shows the number of elements belonging to each annotation (compliance or violation) in each cluster. Note that for most of the clusters, comprising of $90 \%$ of the 5 minute epochs in a trace collected over a month, the vast majority of elements in each cluster correspond to one annotation. In addition these clusters are different from one another. Figure 7 depicts the cluster centroids (with a subset of the metrics) for four of the cluster from Table 4: cluster 4 and 7, that contained only compliance signatures and cluster 1 and 3 , that contained mostly violation signatures. Note that the compliance centroids deem most metrics as not attributed with a violation state (values - 1 for metrics), while the violation centroids deemed some of the metrics as attributed with a violation state (value 1), and others as either irrelevant (value 0 ) or non-attributed. We also see the difference between the centroids of the "violation" clusters (1 and 3) with respect to the metrics that are deemed attributed. Cluster 1 deemed the Database tier CPU utilization (DB1 cpubusy) as attributed but assigned a -1 value for the application server CPU utilization (gbl cpu total util). In contrast, the centroid of cluster 3 deemed the application server CPU as attributed, together with the number of alive processes and active processes on the applica- 

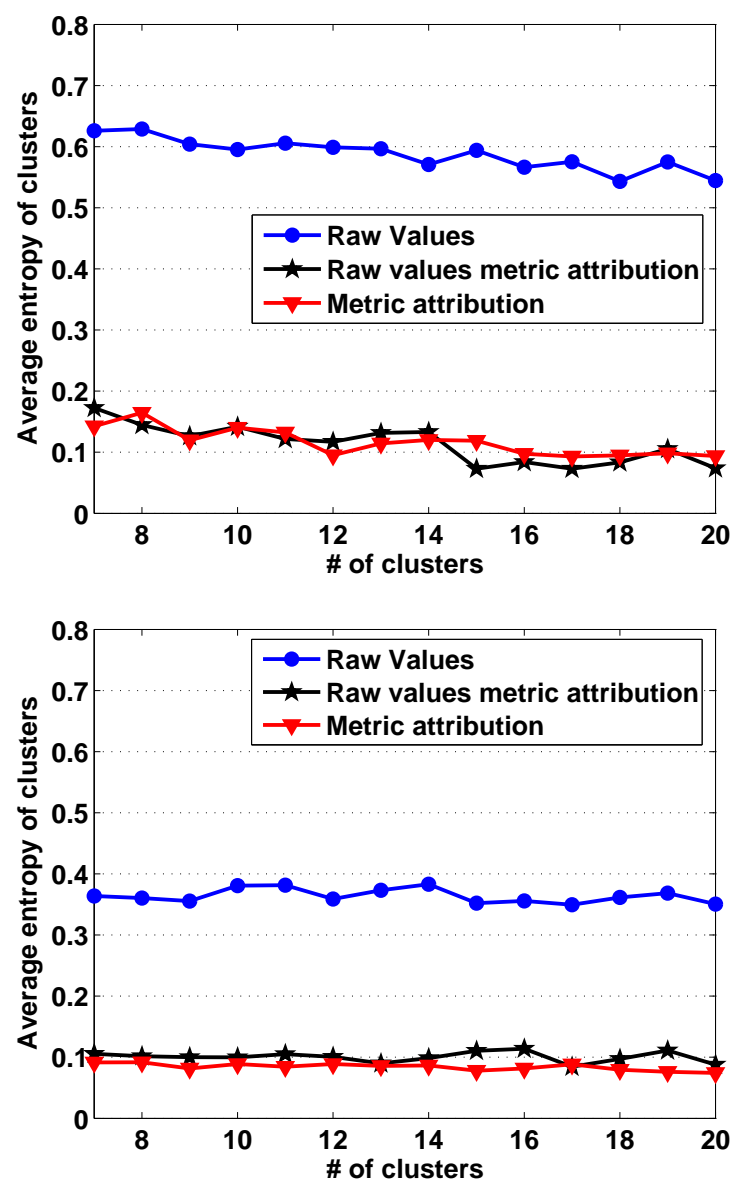

Figure 3: Clustering of the data from RWA. Each graph corresponds to a different server. The signatures relying on information from metric attribution outperform those using only raw values.

tion server. As discussed earlier, most members of cluster 3 were labeled as the IDC problem, which had the symptom of high application server CPU utilization and high number of alive and active processes. These differences point out to the symptoms of the members in each cluster and define the syndrome of a group of signatures.

Given this clustering, identifying recurrent problem is achieved by looking at the time of occurrence of the signatures in each cluster. Figure 8 depicts the instances of cluster 1, 2 and 3 overlaid in time on the performance indicator graph. Cluster 3 is recurrent, and as mentioned earlier, we verified with the IT operators that the periods defined by "Cluster 3" coincided with the manifestation of the IDC problem according to their records. Thus, had they had this tool, they could have easily identified that problem as a recurring one since it presented the same symptoms as provided by the signatures. In addition, the clustering discovered another undiagnosed recurring problem (Cluster 1 ), with the symptom of higher Database CPU utilization (average of approx. 60\% compared to approx. 20\% in most other times), while at the same time all application

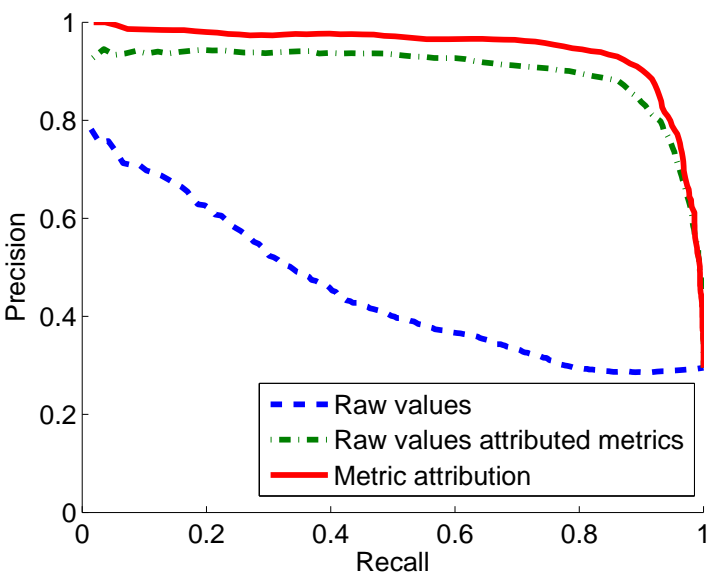

Figure 4: Using data from ETB we see that precision/recall behavior is closest to ideal when metric attribution is used. A method's performance is directly proportional to the area under the curve.

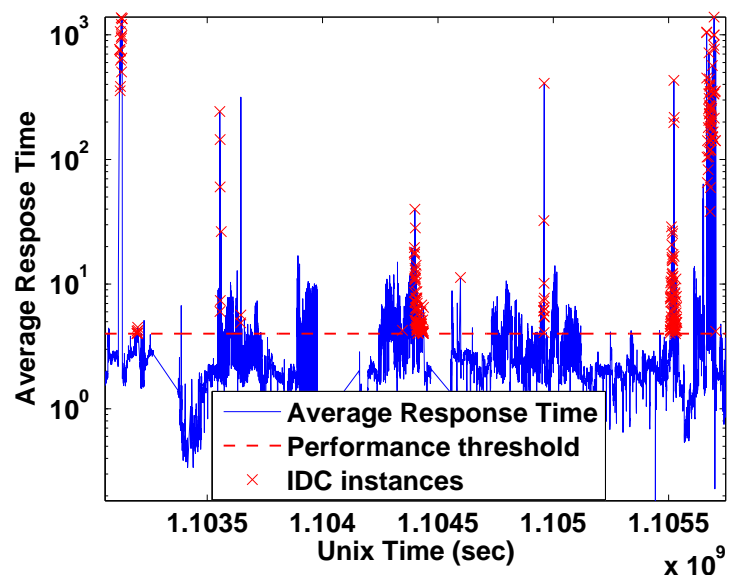

Figure 5: Temporal location of the instances of the IDC problem on one of the Americas machines, overlaid on the reference metric.

server utilization metrics were not attributed, and were in fact normal. This problem remains undiagnosed to this date, and did not appear again in the following months, however, if it appears again, these past instances would be retrieved and perhaps help prioritize finding a solution or the root cause of the problem.

\subsection{Claim 3: Leveraging Signatures Across Sites}

In this section we provide evidence that the signatures collected at various sites and systems can be leveraged during the diagnosis of performance problems. In particular, we show that diagnosis of a performance problem can be aided by querying for similar (or dissimilar) signatures collected at different sites or machines. 


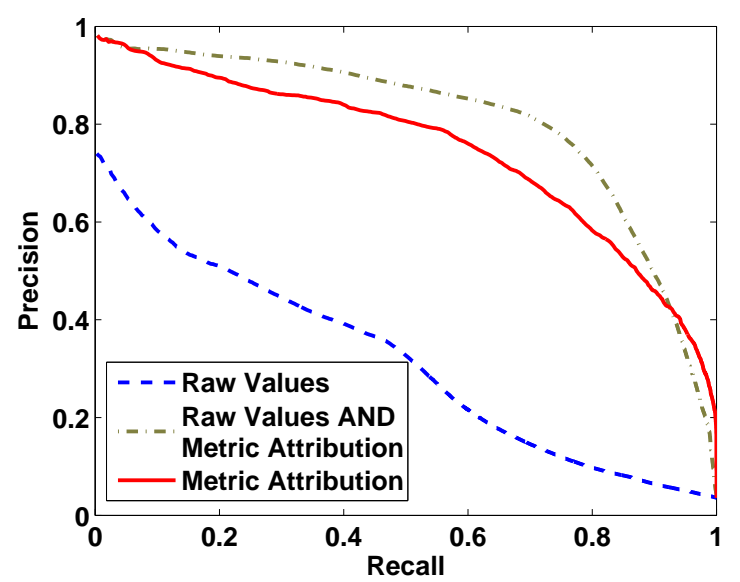

Figure 6: Precision-recall graph for retrieval of the signatures of the IDC issue in the web-service production environment. Methods based on metric attribution outperform the one relying on raw values significantly.

In the process of diagnosing the IDC problem, which was observed on the Americas site, the debugging team investigated whether the same problem occurred in the AsiaPacific region as well. In particular, they hypothesized that it did occur during a failover period on December 18, 2004, in which the transactions from the Americas cluster were being sent to the systems hosting the FT in AsiaPacific(AP). A high percentage of the transactions were violating their SLOs on one of the AP cluster machines during the first 100 minutes of the failover period. The debugging team suspected it was the same IDC problem, and annotated it as such. Our signature database included signatures on traces collected on that day. We then performed the following query: are the signatures annotated as the IDC problem in the Americas region similar to the signatures collected from the AP region? In particular, are those signatures collected during the failover similar to the IDC signatures?

As Figure 9 shows, the result of the query was that the signatures of the AP failover period are very different from the signatures of the IDC problem. Key metrics that were highly attributed in one were not attributed in the other. Of the metrics that were attributed in both, only transaction volume (tt_count) was similar in its attribution signal for the signatures from the two sites.

Upon close inspection of the attributed metrics from one of the AP machines and the transaction mix on that machine, we quickly arrived at a different diagnostic conclusion for the AP problem. Due to failover from the Americas system, AP1 was not only experiencing higher transaction volumes (see Figure 10), but also seeing a type of transaction (call it the XYZ transaction) that it normally does not see. The SQL statements associated with these unusual transactions were not prepared or cached on the AP machines, leading to more database overhead (Figure 12, higher response times, and ultimately SLO viola-

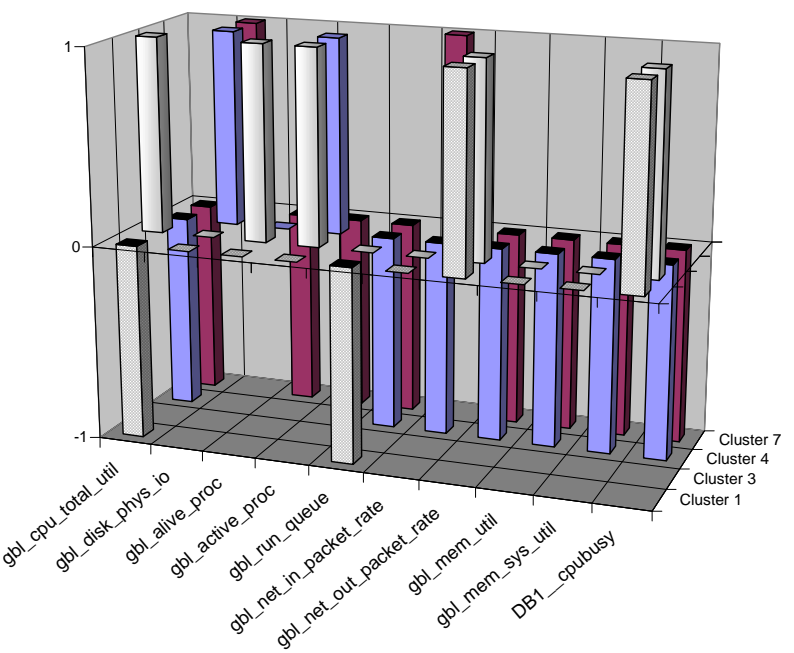

Figure 7: Comparison of the centroid values for four clusters (clusters 1, 3, 4 and 7 from Table 4), two containing mostly compliance signatures (clusters 4 and 7) and two containing mostly violation signatures (clusters 1 and 3 ). Cluster 3 contained mostly signatures of the IDC problem. Note the difference between cluster 1 and cluster 3 in terms of metrics that are attributed and those not attributed.

tion. This diagnosis was accepted by the diagnostics team; the repair consists of priming the database and middleware caches for the new transaction type before a planned failover. As a result of this experience, we were able to replace the false annotation originally provided for that trace data with a new and correct annotation explaining the problem and describing the required repair.

\section{Related Work}

The use of search and retrieval to find similar instances and experiences of faults and performance problems is not new. It is common practice for system operators to use Web search engines to search for the text of error messages or console messages as part of their debugging, but such methods are ad-hoc and imprecise. The work in [15] argues for a machine-readable compact representation of system state in an attempt, among other things, to formalize the recording of instances and that search. Yet to the best of our knowledge our work is the first to propose a concrete technique for constructing the appropriate representation and evaluating its efficacy.

While others have attempted to use low-level metrics to characterize high-level system behavior, we believe that we are the first to propose an automatic method for generating a compact, indexable representation of system state that facilitates syndrome identification and incident clustering. This section surveys recent research on related computer performance diagnosis problems and reviews 


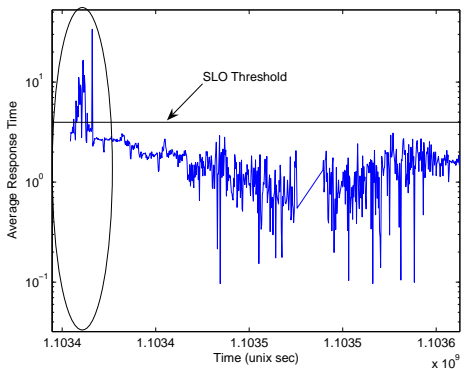

Figure 10: Average response time during the AP1 failover period.

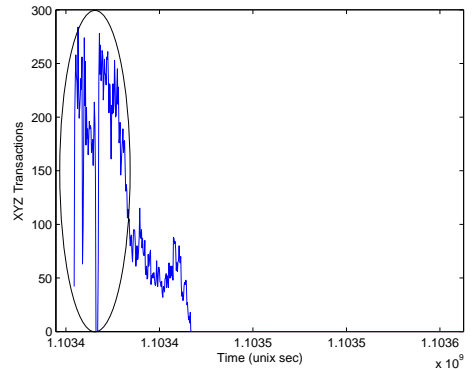

Figure 11: Throughput for the XYZ Transaction during the AP1 failover period. XYZ transactions are usually never seen in AP.

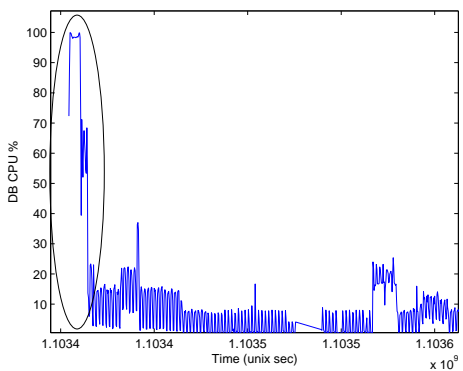

Figure 12: CPU utilization on the DB server was unusually high at the beginning of the failover period. Once the caches are warmed, CPU utilization returns to $20 \%$ or lower.

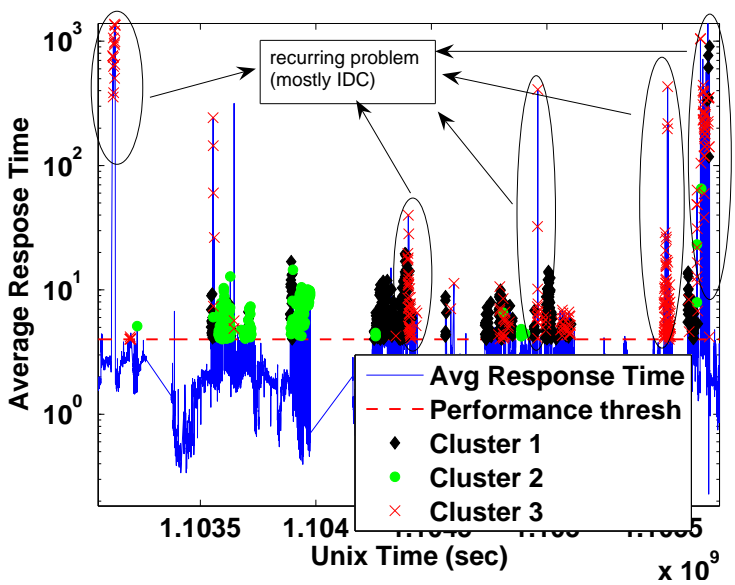

Figure 8: Instances of the three "pure" abnormal clusters (cluster 1, 2 and 3 from Table 4), overlayed on the reference metric . Cluster 3 comprises mostly of the instances annotated as the IDC problem. Cluster 1 is another recurrent problem with the symptom of high Database CPU load and low Application server utilization, while Cluster 2 has memory and disk utilization metrics on the Application server as attributed metrics to the performance problem.

applications of signatures to diagnostic problems in other domains.

Aguilera et al. describe two algorithms for isolating performance bottlenecks in distributed systems of opaque software components [1]. Their "convolution" algorithm employs statistical signal-processing techniques to infer causal message paths that transactions follow among components, which are not assumed to communicate via RPC-like request/reply pairs. At the opposite extreme of this knowledge-lean approach, Magpie characterizes transaction resource footprints in fine detail but requires that application logic be meticulously encoded in "event schema" [2]. Cohen et al. employ statistical pattern classification techniques to identify system utilization metrics that correlate with performance [5].

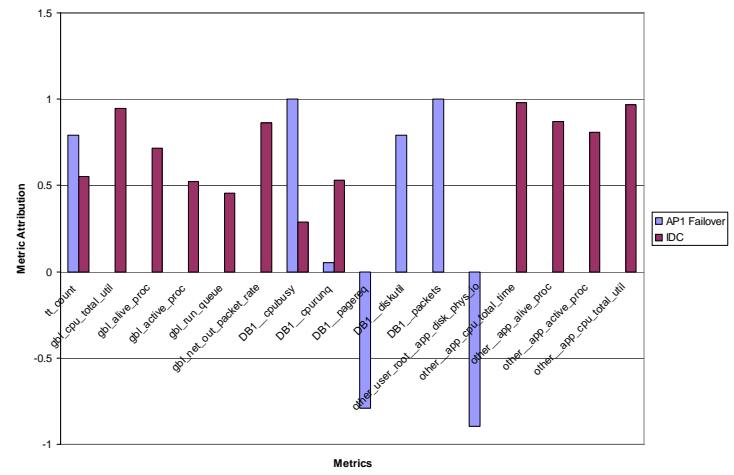

Figure 9: Comparing the signatures from the AP failover period and the IDC problem in the Americas. The bars for each metric show the mean attribution value for the signatures in each period. For metrics where there are not two bars shown, a missing bar means that the metric was not in any model that predicted the violation for this period. Metric names that are not prefixed by "DB1" are from the application server.

Our research differs from these projects in that it is aimed at aiding root cause diagnosis by finding similar problems, rather than bottleneck detection or workload modelling. While our approach does not determine the root cause, finding a previously solved (annotated) problem may provide root cause (or repair action) when the retrieval is accurate. Like Aguilera et al. and unlike Magpie, we assume little knowledge of application structure or logic and we rely heavily on statistical methods. Like Cohen et al. we find that pattern-classification techniques are useful for identifying a small subset of system measurements that are relevant to performance. To us, however, this subset is useful not for its own sake but rather for constructing signatures. For brevity, in this section we do not discuss prior work already summarized in the above papers' excellent literature reviews.

Faults in distributed systems, as opposed to performance problems, are the subject of a large literature. 
For communications networks, whose components have highly constrained and well specified behavior, a wide range of fault localization techniques have been explored [16]. Yemini et al. describe an event correlation (root cause determination) procedure that relies on an extensive library describing each system component's possible faults and the consequences of each fault [21]. These detailed component descriptions are compiled into a codebook that reduces root cause analysis to a simple and efficient task of decoding observed symptoms into the faults that caused them. This approach has been commercialized for communications systems [17] but is inappropriate for arbitrary distributed software because it is infeasible to enumerate the faults and symptoms of arbitrary computer programs. In addition, this approach has no learning or adaptation aspects, which our probabilistic models provide.

The Pinpoint system of Chen et al. analyzes run-time execution paths of complex distributed applications to automatically detect failures by identifying statistically abnormal paths; faulty paths can then aid a human analyst in diagnosing the underlying cause [4]. Kiciman \& Fox describe in greater detail the use of probabilistic context-free grammars to detect anomalous paths in Pinpoint [10]. Our approach shares with Pinpoint the use of statistical techniques, but the instrumentation we require is more readily available and we seek to diagnose performance problems rather than faults.

Jain describes a traditional performance debugging technique to generate visual signatures of performance problems [8]. Popular in the 1970s, "Kiviat graphs" display a handful of utilization metrics in such a way that resource bottlenecks and imbalances assume a distinctive appearance. Like our signatures, Kiviat graphs of different systems (or of different conditions on a single system) invite comparison and facilitate similarity matching. However our signatures differ in several ways from this classic technique: signatures are intended for automated indexing, retrieval, and similarity measurement; they do not rely on human visual inspection; they scale to dozens or hundreds of metrics; and they incorporate application-level performance measures in addition to utilization metrics.

Signatures have been used extensively in virus scanning and intrusion detection [12]. Statistical techniques are often employed to flag anomalous activity automatically, but signatures of malicious behavior are almost always defined manually. Kephart et al. describe a statistical method for automatically extracting virus signatures for a commercial detection product [9].

Redstone et al. advocate automating the diagnosis of user-visible bugs by leveraging the efforts of troubleshooters worldwide [15]. These authors note that such problems have often already been diagnosed and documented, e.g., in newsgroups and vendor bug databases. The real problem is finding the right diagnosis by indexing into a vast disorganized knowledge base. Signatures can help us to realize the vision that Redstone et al. sketch.
Modern systems software, middleware, and styles of application architecture bring obvious benefits but entail substantial costs. Ours is one of several research attempts to preserve the benefits of modern architectural styles while mitigating their problems. We briefly survey three such problems and corresponding research toward solutions.

Layers of modular re-usable components interacting through narrow interfaces allow us to divide and conquer increasingly complex problems at low cost. However they also conceal performance-critical information about each component from its neighbors. For example, conventional operating systems offer strong inter-process fault isolation but suffer side effects including redundant data buffering and copying. The IO-Lite buffering/caching subsystem retains fault isolation while eliminating redundancy [13].

Resource virtualization permits application developers to ignore congestion and scarcity, which they often do to the detriment of performance. The SEDA framework encourages application designers to explicitly address overload and resource scarcity while retaining many of the benefits of virtualization [19].

Finally, decentralized management and geographic distribution allow different organizations to cooperatively serve a global user base, but these trends also diffuse the knowledge required for performance debugging. Aguilera et al. and Cohen et al. confront the opacity of complex modern applications by illuminating performance bottlenecks and correlates of performance in unmodified distributed applications $[1,5]$. Our signature-based syndrome identification methods reduce redundant diagnostic effort across time, geography, and organizational boundaries.

\section{Discussion}

\subsection{On Root Cause Analysis and Diagnosis}

The statistical and pattern recognition techniques underlying the automated extraction of the signatures capture correlation, not necessarily causation. Indeed, as is well known in the statistics and other communities, the ability to infer causation from pure observation is limited and in most cases impossible [14]. By pure observation we mean lack of direct intervention into the system or additional information, coming from human experts regarding the causal relations and paths in the system. In some instances, time information and information about the sequence of events can be used as heuristics to find causal connections. This has been attempted in many domains including this one, most notably in [1]. We leave as future work the inclusion of this kind of information into our approach and the exploration of its utility, although we remark that there is nothing in principle that prevents us from considering "sequences" of signatures or adding time information (including precedence information) into the creation of the signatures and the subsequent analysis. 
It follows from this discussion that we cannot claim (nor have we ever) that the approach advocated in this paper yields a root cause of the problem. Indeed, even with human expert knowledge, root cause analysis is far from easy (recall the example of Section 3.3). Nevertheless, we postulate that offering the capabilities of systematic similarity search and clustering of correlated metrics helps in narrowing down the possible causes and is therefore useful in the diagnosis process. In addition, it may not be necessary to reach a root cause to produce a repair. As difficult as root cause analysis has proven to be over the years, perhaps a more pragmatic approach would be to automatically map the evidence for the faults and metric state to a finite set of possible repair actions.

\subsection{On Annotations, the Real World, and Clustering}

For the scenarios we target-assistance in narrowing down the likely causes of performance/availability problems and the ability to re-use diagnostic efforts across sites as well as across time-we must assume that we start from un-annotated data logs. Our methodology relies on the pragmatic assumption that if our clusters have low entropy, the clusters have done a good job of grouping what would likely be signatures with the same annotation if the data were annotated; this makes clustering useful even in the absence of annotations. This assumption is supported by our results demonstrating that the method we use for signature generation results in both high-quality clustering and excellent precision-recall behavior when we do have annotations. In fact, the clustering was able to recognize $75 \%$ of the IDC cases as belonging to the same cluster.

Similarly, our evaluation of how well our technique can identify recurring problems is really just checking whether we would identify two data points as belonging to the same cluster if they were collected at different points in time-again, whether the clustering and signature-based query results support each other. When they do, we can tentatively conclude that the size of a particular cluster reflects how many violations can be attributed to the "syndrome" that cluster represents; when a human operator investigates further, characterizes and names the syndrome, and describes remediation procedures, these can be permanently stored in the database.

Still, it is a fact of life in the IT trenches that annotations will be scarce and also imperfect. Part of the reason for imperfect annotations on real data results from the reality that the administration of different subsystems or tiers of an application may be delegated to different individuals distributed across the organization, as we experienced when investigating the problem and resolution described in Subsection 4.3. Our experience with other companies running multi-tier applications confirms that there is often no single administrator responsible for understanding the end-to-end paths through the application. One result of this is a frequent lack of clear agreement on what the true cause of a problem is or was: forensic data may be discarded before it's needed, and each operator is typically focused on either debugging or exonerating her/his piece of the system. So un-annotated or imperfectly annotated data should be considered realistic. We hope that the availability of a systematic way to exploit annotations, as proposed in this paper, will encourage a change in best practices. Our acceptance of good clustering and the centroids as a "proxy" for annotated data represents a practical approach when un-annotated data is all that we have at our disposal.

Of course much more monitoring and experience with real data is needed to validate our approach over the long run. The contribution of our present work is proposing and implementing specific methods and illustrating the value of indexing, similarity search, and clustering for diagnosis on real data.

\subsection{Performance Impact of Our Approach}

There are five points where our approach adds computation cost or other overhead that may impact performance considerations: overhead of collecting data, construction of TAN models for metric attribution [5], signature computation, clustering, and retrieval. Our system data is collected by a commercial tool that is widely deployed in industry; the tool is designed to minimize performance impact on the observed system, and at any rate the widespread use of such tools represents a sunk cost. Updated system data is coalesced and reported periodically, generally in 1 or 5 minute epochs. Building and maintaining an ensemble of TAN models takes 5-10 seconds with our code, so it is practical to apply the ensemble algorithm to system data in real time. In our prototype implementation in Matlab, given an ensemble with approximately 41-67 models (generated using one month of system data), it takes about $200 \mathrm{~ms}$ to compute a signature for one epoch. Using the $k$-medians algorithm (with $k=10$ ) to cluster 7507 signatures (about one month when using 5-minute epochs) takes less than 10 seconds. Finally, retrieving the top 100 matching signatures from a database of 7700 signatures takes less than one second. We conclude that signature generation can proceed in real time, and analysis with clustering or retrieval is fast enough to be done at will.

\section{Conclusions}

A particularly relevant result we showed is that simply recording the values of raw system metrics does not provide an effective way to index and retrieve syndrome data: a more sophisticated way of generating "signatures" is required. We showed the efficacy of one particular way of doing this using previous work on using ensembles of Bayesian networks to determine which low-level metrics are "implicated" in particular situations and using a list of those metrics (but not their values) as the signature. We 
showed that clustering and retrieval using signatures allows diagnosticians to leverage the results of past work and identify similar or recurring problems, even when no problem annotations or application-specific knowledge are available. Indeed, even our prototype implementation, under these circumstances and working with un-annotated data, was helpful in correcting a misdiagnosis as well as correctly classifying a recurrent problem, procedures that required the exchange of 80 pages of notes among administrators to perform manually.

However, in the large, we are not necessarily advocating for our particular choices of signature construction, clustering, similarity metrics, etc. Indeed, we believe that there is room for improvement and further study in any of these issues. Rather, we are advocating the idea that being able to automatically distill the essence of an observed undesirable behavior, and index a machine-manipulable representation of that essence for troubleshooting, is a logical next step for systems research on automation that will facilitate scaling and increase the availability of our systems. In addition, it will enable a systematic way of capturing knowledge and expertise from operators (through annotations of diagnoses and repair actions) that can be leveraged by other operators across geography and across time.

\section{References}

[1] M. K. Aguilera, J. C. Mogul, J. L. Wiener, P. Reynolds, and A. Muthitacharoen. Performance debugging for distributed systems of black boxes. In Proc. 19th ACM SOSP, 2003.

[2] P. Barham, A. Donnelly, R. Isaacs, and R. Mortier. Using Magpie for request extraction and workload modelling. In Proc. 6th USENIX OSDI, Dec. 2004.

[3] G. Candea, S. Kawamoto, Y. Fujiki, G. Friedman, and A. Fox. A microrebootable system - design, implementation, and evaluation. In Proc. 6th USENIX OSDI, San Francisco, Dec. 2004.

[4] M. Chen, E. Kicıman, E. Fratkin, E. Brewer, and A. Fox. Pinpoint: Problem determination in large, dynamic, Internet services. In Proc. International Conference on Dependable Systems and Networks, pages 595-604, Washington, DC, June 2002.

[5] I. Cohen, M. Goldszmidt, T. Kelly, J. Symons, and J. S. Chase. Correlating instrumentation data to system states: A building block for automated diagnosis and control. In Proc. 6th USENIX OSDI, San Francisco, CA, Dec. 2004.

[6] R. O. Duda, P. E. Hart, and D. G. Stork. Pattern Classification. Wiley, second edition, 2001.

[7] D. Jacobs. Distributed computing with BEA WebLogic server. In Proceedings of the Conference on Innovative Data Systems Research, Asilomar, CA, Jan. 2003.

[8] R. Jain. The Art of Computer Systems Performance Analysis. Wiley-Interscience, New York, NY, 1991.

[9] J. O. Kephart and W. C. Arnold. Signatures. In Proc. 4th Virus Bulletin International Conference, 1994. http://www.research.ibm.com/antivirus/ SciPapers/Kephart/VB94/vb94.html.
[10] E. Kiciman and A. Fox. Detecting application-level failures in component-based internet services. IEEE Transactions on Neural Networks, Spring 2005.

[11] D. Mosberger and T. Jin. httperf-a tool for measuring web server performance. http://www.hpl.hp.com/ personal/David_Mosberger/httperf.html.

[12] B. Mukherjee, L. T. Heberlein, and K. N. Levitt. Network intrusion detection. IEEE Network, 8(3):26-41, May 1994.

[13] V. S. Pai, P. Druschel, and W. Zwaenepoel. IO-Lite: A unified I/O buffering and caching system. ACM Trans. Comput. Sys., 18(1):37-66, Feb. 2000.

[14] J. Pearl. Causality: Models, Reasoning, and Inference. Cambridge University Press, 2000.

[15] J. A. Redstone, M. M. Swift, and B. N. Bershad. Using computers to diagnose computer problems. In Proc. HotOS $I X$, pages 91-96, May 2003.

[16] M. Steinder and A. S. Sethi. A survey of fault localization techniques in computer networks. Science of Computer Programming, 53:165-194, 2004.

[17] System Management Arts (SMARTS) Inc. Automating root cause analysis, 2001. http: / / www . smarts . com.

[18] The Open Group. Application Response Measurement (ARM) 2.0 Technical Standard, July 1998. http: //www. opengroup.org/onlinepubs / $009619299 /$ toc.pdf.

[19] M. Welsh, D. Culler, and E. Brewer. SEDA: An architecture for well-conditioned, scalable Internet services. In Proc. 18th ACM SOSP, 2001.

[20] I. H. Witten and E. Frank. Data Mining: Practical Machine Learning Tools and Techniques with Java Implementations. Academic Press, 2000.

[21] S. A. Yemini, S. Kliger, E. Mozes, Y. Yemini, and D. Ohsie. High speed and robust event correlation. IEEE Communications Magazine, pages 82-90, May 1996.

[22] S. Zhang, I. Cohen, M. Goldszmidt, J. Symons, and A. Fox. Ensembles of models for automated diagnosis of system performance problems. In DSN, 2005. 